This item was submitted to Loughborough's Research Repository by the author.

Items in Figshare are protected by copyright, with all rights reserved, unless otherwise indicated.

\title{
Securing DICOM images based on adaptive pixel thresholding approach
}

PLEASE CITE THE PUBLISHED VERSION

https://doi.org/10.1109/CBMS.2018.00056

PUBLISHER

IEEE

VERSION

AM (Accepted Manuscript)

PUBLISHER STATEMENT

(c) 2018 IEEE. Personal use of this material is permitted. Permission from IEEE must be obtained for all other uses, in any current or future media, including reprinting/republishing this material for advertising or promotional purposes, creating new collective works, for resale or redistribution to servers or lists, or reuse of any copyrighted component of this work in other works.

\section{LICENCE}

All Rights Reserved

\section{REPOSITORY RECORD}

Natsheh, Qamar, Ana Salagean, and Eran Edirisinghe. 2018. "Securing DICOM Images Based on Adaptive Pixel Thresholding Approach”. figshare. https://hdl.handle.net/2134/12115605.v1. 


\title{
Securing DICOM images based on adaptive pixel thresholding approach
}

\author{
Qamar Natsheh, Ana Sălăgean, Eran Edirisinghe \\ Department of Computer Science \\ Loughborough University \\ Loughborough, Leicestershire, UK \\ q.natsheh@lboro.ac.uk
}

\begin{abstract}
This paper presents a novel efficient two-region Selective encryption approach that exploits medical images statistical properties to adaptively segment Digital Imaging and Communications in Medicine (DICOM) images into regions using thresholding mechanism in the spatial domain. This approach uses adaptive pixel thresholding, in which thresholds for same DICOM modality, anatomy part and pixels intensity's range were extracted off-line. Then, the extracted thresholds were objectively and subjectively evaluated to select the most accurate threshold for the correspondent pixel intensity range. In the on-line phase, DICOM images were segmented into a Region Of Interest (ROI) and a Region Of Background (ROB) based on their pixels intensities using the adopted thresholds. After that, ROI was encrypted using Advanced Encryption Standard (AES), while ROB was encrypted using XXTEA. The main goal of the proposed approach is to reduce the encryption processing time overhead in comparison with the Naïve approach. The proposed approach aims to achieve a trade-off between processing time and a high level of security. The encryption time of the proposed approach can save up to $60 \%$ of the Naïve encryption time for DICOM images with smallmedium ROI.
\end{abstract}

Keywords-DICOM; medical images; image segmentation; AES; XXTEA; selective encryption; processing time

\section{INTRODUCTION}

The recent advancements in communication technologies have enabled e-health/m-health industry to remotely monitor the patients' conditions, therefore enhancing the healthcare services [1]. Various types of medical images are produced using different DICOM imaging modalities, which are sent via public networks to healthcare centres to facilitate ongoing medical diagnosis processes [2].

The transmission of the very sensitive collected medical data/images over the public network; e.g. Internet, is vulnerable to many security threats. To address this problem, several techniques have been introduced, such as encryption algorithms, which encrypt the entire pixel data, header data or both. However, security applications, such as traditional encryption approaches; are not suitable for bulk data as medical images; due to their complexity and redundancy nature of images' pixels data [3]. In addition to that, workstations and handheld devices have limited resources. Many approaches have been proposed to overcome these limitations; such as selective encryption. The concept of selective encryption is presented to overcome this limitation, where the medical image's pixel data is partially encrypted using sophisticated encryption algorithms, thereby reducing the complexity overhead.
Typically, in full (Naive) and selective image encryption schemes the original image is viewed as a $2 \mathrm{D}$ array of pixels. Full image encryption is realized by treating the 2D array of pixels as a 1D textual bitstream. Therefore, any conventional cryptographic technique can be applied directly to encrypt the entire bitstream. While in selective encryption, visual image data must be segmented into a ROI and ROB. This segmentation can be performed by visual inspection methods (manual) to determine the most meaningful optical parts of the image, according to various aspects, such as boundaries or object's background. Then, the selected segments will be secured using encryption, while other parts of the image are left unencrypted.

In this paper, we propose Selective encryption approach based on adaptive pixel thresholding technique, where segmented images are secured using AES for informative pixels and XXTEA for less informative pixels. The main goal of the proposed approach is to introduce an efficient selective encryption that reduces the processing time overhead of the DICOM encryption process in comparison with Naïve approach; where all image pixels are encrypted using AES, while maintaining security of the pixel data. As prat 15 in DICOM standard recommended to secure DICOM images' pixel data prior to exchange DICOM data [31].

The segmentation process in this paper is performed in the off-line stage, to extract relevant DICOM pixels thresholds. Then, in the on-line stage, extracted thresholds are used to identify regions for selective encryption. After that, segmented regions are encrypted using different encryption algorithms based on their importance. The novelty of this work that it efficiently secures DICOM images' pixels data automatically and selectively in the spatial domain; as most of the DICOM images selective encryption research performed in the spatial domain depends on manual selection of informative data.

The rest of the paper is structured as follows: The background elaborated in Section II. Section III presented the proposed two-region Selective encryption approach. Experimental results and discussion are shown in Section IV. Section $\mathrm{V}$ presented the conclusion and future research directions.

\section{BACKGROUND}

In general, selective encryption approaches can be performed in the spatial [4] - [8] or the frequency domain [9][10]. Most of the conducted research on selective encryption was in the frequency domain. However, spatial domain selective encryption is simple and does not require any transformation of pixel intensity into other domains. 
In the spatial domain, Lamri et al. [5] proposed the use of $\mathrm{N}$-grams to detect objects in the medical images and to encrypt only the selected region. However, the proposed approach left other regions without encryption; also, the overall shape of the anatomy part is preserved. Another important drawback that $\mathrm{N}$-gram is an iterative based approach which increases the processing time overhead. Mbarek et al. [4] suggested the use of K-means to segment the DICOM image into subsets then store them in distributed cloud systems where each subset was stored in a different cloud. In this method, the image was secured as it is not readable, and its' subsets were stored on different physical cloud systems. The main drawback of this method that it requires an expensive infrastructure and it requires additional mechanisms to combine distributed subsets and retrieve medical images. In both works [5], [4] the selection process was executed in the spatial domain as segmentation was performed directly on the pixel intensity. However, both methods did not encrypt selected regions using recognised encryption algorithm.

An adaptive selective encryption algorithm was introduced in [6] - [8]. The main idea of adaptive selective encryption algorithm is to encrypt DICOM images using different encryption algorithms and different keys' lengths based on required security level and processing time. The proposed method is promising for speeding up the encryption time and marinating the security level. However, reducing encryption time and achieving good robustness are contradictory to each other and the proposed method must achieve a trade-off between robustness and short encryption time. Moreover, Mahmood et al. [6] utilised Genetic Algorithm (GA) to determine the key size, number of regions and the importance of regions. GA is hard to be tuned and it requires many parameters and functions to be reset before using GA. Moreover, GA does not guarantee the optimal solution. Mahmood et al. suggested additional permutation to overcome this limitation.

To sum up, spatial domain selective encryption approaches in the literature require user input to perform segmentation (manual segmentation) and does not exploit any statistical properties of medical images. In this work, we present an automatic Selective encryption approach in the spatial domain that aims to reduce the processing time overhead of encryption algorithms and adaptively threshold DICOM images based on their modalities, anatomy part and pixel intensity range.

\section{PROPOSED APPROACH}

In order to achieve the goal of reducing the processing time overhead for encrypting DICOM images' pixel data (greyscale), this data must be separated into segments based on their importance, in which informative segment (ROI) was encrypted using sophisticated encryption method, while less informative segment (ROB) was encrypted using lighter encryption method.

In general, medical images from same modality and same anatomy part tend to have the same distribution [11], [16]. To the author knowledge, this statistical propriety has not been exploited in any medical images segmentation technique before [12], [13]. Accordingly, this paper proposes a Selective encryption approach, which employs this statistical property to adaptively segment DICOM images using pixel thresholding. In other words, DICOM images from same distribution (same anatomy part and pixel intensities range) have the same threshold regardless of the patient, imaging devices, imaging devices settings, etc.

The proposed approach executed in two stages; which are off-line thresholds extraction and on-line selective encryption; as shown in Fig. 1. The off-line thresholds extraction stage involves sorting same DICOM images modality and anatomy part based on their pixels' intensities ranges. Then, pixels thresholds were determined using accurate segmentation methods (e.g. clustering methods). Then, extracted thresholds were evaluated on testing DICOM dataset to identify the correspondent thresholds for the on-line stage over different pixels' intensities ranges.

In the on-line stage, the adopted thresholds were employed for automatic pixel thresholding, as medical images from same anatomy part and same modality have similar distribution [16], extracted thresholds can be used adaptively to segment medical images from same statistical group, and then segmented parts were selectively encrypted to reduce the processing time overhead. It is possible to encrypt ROI and preserve ROB without encryption. However, it is recommended to encrypt $\mathrm{ROB}$ as it might expose some important information (perceptual security). Also, encrypting both ROI and ROB maintain the coherence of the encrypted image.

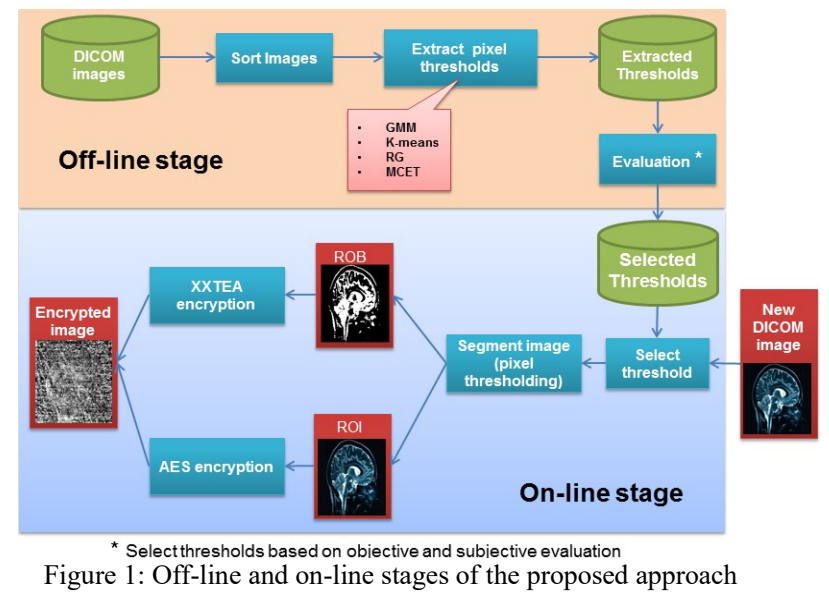

A. Regions selection mechanism: Segmentation

In general, segmentation methods can be classified into three main groups, which are machine learning techniques, deformable models, and fundamental techniques (thresholding and statistical based methods) [14]. However, advanced segmentation methods such as machine learning and deformable methods require additional processing time. Nevertheless, fundamental segmentation techniques are simple methods; such as thresholding; because of their intuitive properties, the simplicity of implementation and processing speed. 
Thresholding algorithms include three methods, namely [15]; pixels-based, edge-based and region-based methods. Pixel thresholding method is the fastest amongst other methods and it was adopted in this research. However, it requires a pre-defined threshold which makes this method impractical for multimedia images, due to the huge variations amongst multimedia images (e.g. shape, intensities, objects, etc.). Further, medical images' variations follow same distributions, which mean that medical images from same modality, same anatomy part and same pixel intensity range have almost the same distributions [16]. This fact facilitates the use of pre-defined thresholds. As, the encryption approach must be automatic; so an automatic segmentation technique that can segment different types of DICOM images is needed. Therefore, pixel thresholds were determined off-line using accurate segmentation methods. Then, for on-line stage, thresholds were changed adaptively based on image modalities, anatomy part and pixels intensities ranges.

\section{B. Off-line thresholds extraction stage}

To extract accurate and reasonable thresholds for different DICOM modalities, anatomy parts and pixel ranges intensities, DICOM images must be segmented using advanced segmentation methods. In this research, machine learning methods (i.e. K-means [17] [18] and Gaussian Mixture Model (GMM) [19]), region-based segmentation method (i.e. Region Growing (RG) [20] [21]) and automatic thresholding method (i.e. Minimum-Cross Entropy Threshold (MCET) [22]), were utilised. After that, thresholds were extracted from the segmented images in the off-line stage. Then, extracted thresholds were subjectively and objectively evaluated on testing DICOM dataset to identify the most reliable thresholds for the on-line stage.

In the off-line stage, images from different modalities where sorted into different groups based on their intensities ranges. For this work, images were grouped into three main groups; these groups were identified based on the mean value $\left(\mu_{\max }\right)$ and Standard Deviation $\left(\sigma_{\max }\right)$ of the maximum pixels intensities for all images in a given DICOM modality (training dataset). Fig. 2 shows the maximum pixel intensities of 200 $\mathrm{CT} /$ Head DICOM images; the first group (pink) is images with maximum pixels' intensities vary between $\left[0, \mu_{\max }-\right.$ $\sigma_{\max }$ ) (range 1). The second group (blue) is images with maximum pixels' intensities vary between $\left[\mu_{\max }-\sigma_{\max }\right.$, $\left.\mu_{\max }+\sigma_{\max }\right)($ range 2 ). The last group (yellow) contains images with maximum pixels' intensities larger than $\mu_{\max }+$ $\sigma_{\max }$ (range 3).

These ranges were identified for every DICOM modality and anatomy part. The aforementioned segmentation approaches were utilised over every range separately to identify its corresponding threshold. It is worth here to mention that the segmentation accuracy can be improved by increasing the number of intensities ranges around the mean value of the maximum intensities. Finally, the extracted thresholds for different DICOM modalities, human anatomy parts and ranges were objectively and subjectively evaluated, then adopted thresholds were saved in data structure (as shown in Fig. 1) to segment DICOM images in the on-line phase.

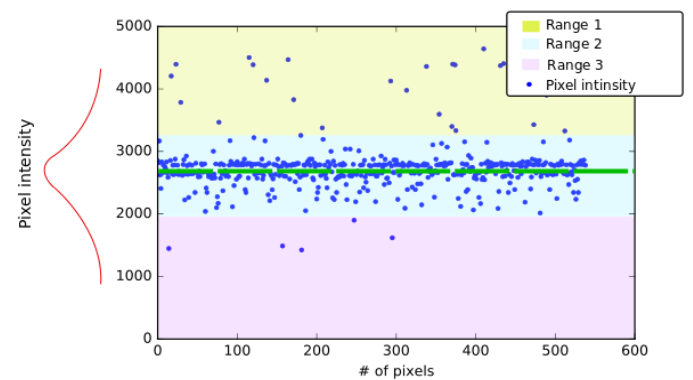

Figure 2: Pixel intensities ranges

\section{On-line pixel thresholding and encryption}

After extracting the suitable thresholds for each modality, anatomy part and pixels intensity ranges, extracted thresholds can be used on-line to segment images based on their category. Then, ROI and ROB can be selectively encrypted to reduce the processing time overhead, in which ROI will be encrypted using AES (high security and adopted by National Electrical Manufacturers Association (NEMA)) [23] [24], while ROB will be encrypted using XXTEA as a lighter encryption algorithm, as shown in Fig. 1. In addition to the ROI and ROB, a segmentation map was generated from this stage, which identifies the ROI and ROB pixels' locations in the original image. This segmentation map is very important to reconstruct the decrypted image. Hence, it must be saved in the DICOM header and secured with the header in accordance with the DICOM standard [31] [32].

\section{EXPERIMENTAL RESULTS}

\section{A. Datasets and Experimental Setup}

To extract accurate thresholds and evaluate the proposed two-regions Selective encryption approach, a large dataset that contains DICOM images of different modalities (Computed Tomography (CT), Magnetic Resonance (MR), Mammography, X-Ray) and different parts of the human body (Ankle and Foot, Head and Brain, Shoulder, Knee, Pelvis, Hip, Chest and Breast) were used to determine thresholds for different DICOM modalities [25] - [29].

All segmentation techniques were trained on $600 \mathrm{CT}, 464$ MR, 27 X-Ray and 16 Mammographic DICOM images to obtain pixel thresholds that can be used to segment medical images in the on-line stage. Also, for evaluation purposes another testing dataset was used; which consists of 30 CT, 184 MR, 106 X-Ray and 53 Mammography DICOM images.

In K-means and GMM cases; two different dataset's arrangements were used to estimate thresholds. In the first dataset, the pixels of all images were combined (pixels dataset) in one feature array, then pixels in this dataset were clustered into two groups (ROI and ROB). In the second approach, regions were identified individually for each image in the given dataset (image dataset). Then thresholds' average value of all images in the image dataset for a specific modality was reported. The reason behind using these two data structures (pixels and images datasets) was to test which data structure can give the best generalisation. 
Additionally, RG and MCET were trained based on the individual images' pixels (image dataset), like the later approach in the K-means and GMM case since RG and MCET are image-based methods while GMM and K-means are generic clustering approaches.

After identifying ROI and ROB of the given dataset using aforementioned segmentation methods, statistical properties of the ROB were utilised to extract set of thresholds for a given modality. These statistical values include; maximum (MAX), mean (MEAN), standard deviation (STD) and median (MEDIAN) of ROB pixels' intensities. In the next section, the extracted thresholds were tested and evaluated using the testing dataset to evaluate and select the most appropriate threshold.

It is worth here to mention that the experiments were conducted in a Cython/Python environment running on a ThinkPad T430s machine (Intel CORE i5, 8.00 GB RAM at 2.6 GHz with Ubuntu 14.04).

\section{B. Segmentation results}

Segmented testing images based on the extracted thresholds (MAX, MEAN, STD+M and MEDIAN) were subjectively and objectively evaluated for every modality. Then, segmentation quality of different extracted thresholds and different segmentation methods were compared. The comparisons between segmentation methods were based on a subjective and objective analysis, which give more clues to identify the suitable threshold for every modality, every human anatomy part and every pixel intensity range. The subjective analysis relies on human naked eye inspection and it varies based on the human knowledge and experience. While, objective analysis is a set of deterministic metrics that measures the segmentation quality based on some statistical measurements.

\section{1) Subjective analysis}

The Subjective analysis is the most widely used evaluation method, in which the segmentation results are examined by a human evaluator. The drawback of such evaluation method is that visual or qualitative evaluation is inherently subjective. Subjective evaluation may vary from one observer to another because each human has her/his own specific standards for evaluating segmented images. Moreover, the evaluation results can be affected by the order of the assessments process of the segmentation results. Hence, extracting a neutral evaluation of a segmentation algorithm is a challenging task, as it requires a large intensive visual evaluation study.

2) Objective analysis

By a close look at the DICOM image segmentation problem, it can be seen as a clustering problem (unsupervised learning). Thus, the aforementioned extracted thresholds can be objectively evaluated using clustering evaluation metrics. The metrics used to evaluate the extracted thresholds were Discrepancy Error $\left(\mathrm{D}_{\mathrm{er}}\right)$, Normalised Uniformity (NU), Calinski-Harabz Index (CHI) and information gain (Entropy). The $\mathrm{D}_{\text {er }}$ indicates the difference between the segmented object and the original image, higher $D_{\text {er }}$ indicates a good segmentation in noisy images; while it is almost zero for nonnoisy images. The second metrics is NU that indicates the consistencies of regions ( 1 indicates the best consistency). The
$\mathrm{CHI}$ index is given as the ratio of the between-segments dispersion mean and the within-segment dispersion, and a higher $\mathrm{CHI}$ value relates to thresholds that define better segments. Table I shows the evaluation metrics results for CT/Head segmented images using thresholds extracted based on K-means (image dataset) amongst range 2 thresholds. Accordingly, the best threshold in this table is compared with the best threshold extracted based on other segmentation methods for the same DICOM modality.

TABLE I: Objective analysis results of extracted thresholds for CT/Head

\begin{tabular}{cccccc}
\multicolumn{6}{c}{ (Range 2) based on K-means (image dataset) } \\
$\begin{array}{c}\text { Threshold } \\
\text { Type }\end{array}$ & CHI & $\mathbf{D}_{\text {er }}$ & NU & $\begin{array}{c}\text { ROB } \\
\text { Entropy }\end{array}$ & $\begin{array}{c}\text { ROI } \\
\text { Entropy }\end{array}$ \\
\hline MAX & $\begin{array}{c}2.26 \mathrm{E}+ \\
06\end{array}$ & $\begin{array}{c}3.74 \mathrm{E}+ \\
06\end{array}$ & $\begin{array}{c}0.99962 \\
2\end{array}$ & 1.87106 & 8.5241 \\
& 06 & 35973. & 0.99999 & 0.22968 & \\
MEAN & 185500 & 6 & 9 & 5 & 8.23091 \\
& & 6 & 0.99997 & 0.94281 & 8.33467 \\
STD+M & 342615 & 955491 & 3 & 6 & \\
& & 3329.6 & 1 & 0.09467 & 8.25204 \\
MEDIAN & 179923 & 2 & 1 & 88 & \\
\hline
\end{tabular}

For each DICOM modality and pixel intensity range the threshold was adopted, based on the objective and subjective evaluation. In general, it was noticed that K-means dominated all other methods in accuracy, except images with small ROI size (with ROI/Image size ratio of $\leq 20 \%$ ) that were segmented efficiently using thresholds extracted based on GMM, while RG was the most accurate for the large ROI size (with $\mathrm{ROI} / \mathrm{image}$ ratio of $\geq 75 \%$ ). Also, it was observed that MEAN and MEDIAN thresholds; based on all segmentation methods, were robust against noise.

\section{Processing time: Naïve vs proposed approach}

The adopted thresholds based on the previous step were used to segment DICOM images, and then they were encrypted using the Selective proposed encryption approach. In which, DICOM testing dataset were firstly segmented into ROI and ROB. Then, ROI was encrypted using AES and XXTEA cipher was used to encrypt ROB. The AES was used with Counter mode of operation and 256 bits key length, while XXTEA was used with 128 bits key length. Finally, the encryption time of the proposed approach was compared with the encryption time of the Naïve approach.

Fig.4 shows the saved time using the proposed approach with respect to the Naïve approach against the ratio of ROI to image size. It was noticed that the proposed approach had shorter processing time (segmentation and encryption time) in comparison with Naïve encryption approach for DICOM images with ROI size less than $\sim 55 \%$ of the image size, for those images the proposed approach can achieve up to $60 \%$ saved time. Also, it was noticed that at $\sim 55 \%$ ROI size ratio; CPU processing time variation might outpace the saved time by the proposed approach as in MR DICOM images. What is more, the proposed approach saving time was almost zero for DICOM images with large ROI size (approx. $\geq 64 \%$ ). Furthermore, for images where ROI sizes are equal to the image size, the proposed approach had the longest processing time in comparison with Naïve approach. 


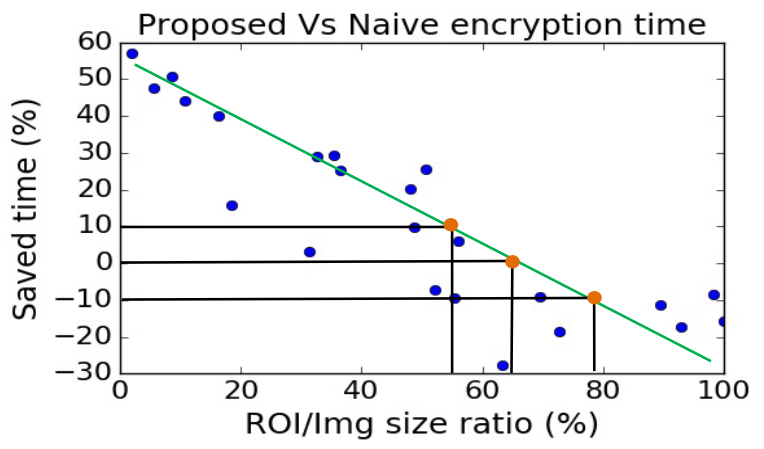

Figure 3: Saved encryption time using proposed approach vs ROI to image size ratio with respect to Naïve approach

From Fig. 3, we can notice that the proposed approach saved less time in large ROI like X-ray and Mammography images than small ROI like CT-Foot images. Moreover, DICOM images with very large ROI had negative saved time, which means Naïve encryption approach was faster in these cases. In general, it was noticed that proposed approach had shorter processing time when the ROI ratio to the image size is less than $(\sim 55 \%)$. In addition, very small DICOM images, i.e. MR/Shoulder, had a very short encryption time regardless of the encryption method (Naïve or Selective) as they have very small ROI, and the time variation of the computer processor might be larger than the encryption time.

\section{Cryptanalysis}

Image encryption requires cryptographic security, against cryptography attacks, and perceptual security to ensure that encrypted images are not perceptually informative [30]. Many metrics can be utilised to evaluate the proposed encryption approach that can indicate its robustness against different types of attacks. The ability of the proposed approach to diffuse the original image can be evaluated using Mean Absolute Error (MAE), Number of Pixels Change Rate (NPCR), image Entropy and Peak Signal Noise Ratio (PSNR). Hence, encrypted medical image must be considerably different from the original image to avoid a ciphertext attacks. The efficiency of the substitution process can be evaluated using Histogram, Entropy and Normalised Correlation.

For cryptanalysis purposes, 15 DICOM CT/Head images were segmented, encrypted and then analysed using cryptanalysis metrics. The pixel bit depth for these DICOM images was 16. Fig. 4 illustrates selected CT/Head DICOM image before and after encryption. The encryption/decryption time of this image using Naïve vs Selective approaches is exhibted in Table II, in which the encryption time of the proposed approach includes segmentation time, while the decryption time includes the time required for image reconstruction based on the segmentation map. Also, cryptanalysis values, for the $15 \mathrm{CT}$ DICOM images, are shown in Table III.

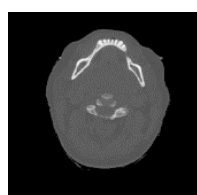

(a)

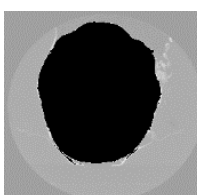

(b)

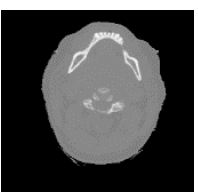

(c)

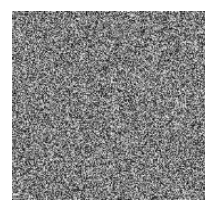

(d)
Figure 4: Selective image encryption: (a) original image , (b) ROB image,

(c) ROI image, (d) encrypted image

TABLE II: CT/Head Encryption/Decryption time

\begin{tabular}{lcc}
\hline & Naïve approach & Proposed approach \\
\hline Encryption time $(\mathrm{sec})$ & 0.013447 & 0.007515 \\
Decryption time $(\mathrm{sec})$ & 0.019083 & 0.007906 \\
\hline
\end{tabular}

Fig. 5 illustrates a flat histogram of the CT/Head encrypted DICOM image. That indicates equal distribution and randomness. Hence, the attacker cannot predict enough information to execute statistical attack.

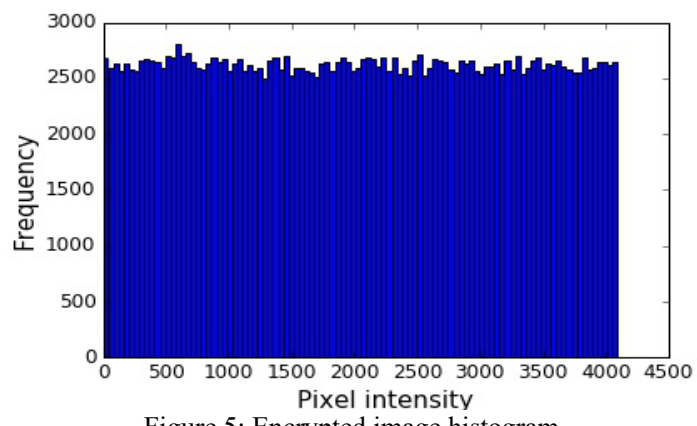

Entropy is a measure of the uncertainty present in the encrypted image. As the bit depth of the DICOM datasets used in this paper is 16 , the entropy value varies between 0 16 bits/pixel. The average entropy for the encrypted CT/Head images was $\sim 12$ bits/pixel which is an effectiveness indication of the proposed approach. Also, the very low correlation value $\sim 0$ indicates the proposed approach immunity to frequency and statistical attacks.

The proposed approach shows a very good diffusion property as MAE and NPCR show a large difference between the encrypted and original image. The encrypted image PSNR value with respect to the original image was $29.16 \mathrm{~dB}$, which shows that the encrypted and original images were greatly different from each other.

TABLE II: Cryptanalysis metrics

\begin{tabular}{cc}
\hline Metrics & Value \\
\hline MAE & 1954.63 \\
NPCR & $99.98 \%$ \\
PSNR & $29.16 \mathrm{~dB}$ \\
Entropy & $11.99 \mathrm{bits} /$ pixel \\
Correlation & 0.0007213 \\
\hline
\end{tabular}

\section{CONCLUSION AND FUTURE DIRECTIONS}

This paper proposed a two-region DICOM image Selective encryption approach. The presented approach was evaluated from the processing time point of view, and encryption robustness using different cryptanalysis metrics. 
The selection process for the proposed approach was based on threshold segmentation in the spatial domain, where regions were identified based on pixels' intensities. The thresholds were extracted off-line using K-means. GMM, MCET and RG for different DICOM modalities, different human anatomies and different pixels intensities ranges. Medical images are different from multimedia images, in the sense that medical images from same anatomy part for different patients have the same statistical distribution, regardless of the imaging device settings. Hence, thresholds extracted for certain anatomy part and within known pixel intensity range can be used to segment images from the same part.

The proposed approach can save time up to $60 \%$ in comparison with Naïve encryption approach for DICOM images with small-medium ROI size. However, the saved time using the proposed approach for images with large ROI (approx. $\geq 55 \%$ ) was very small, where saving time was varying between $[10,-30] \%$. Based on this observation, the proposed approach will be extended into multi-regions Selective encryption approach to increase the saved time for images with ROI larger than $\sim 55 \%$ with respect to DICOM image size.

\section{REFERENCES}

[1] Mildenberger, P., Eichelberg, M. and Martin, E., 2002. Introduction to the DICOM standard. European radiology, 12(4), pp.920-927.

[2] Muhammad, K., Sajjad, M. and Baik, S.W., 2016. Dual-level security based cyclic 18 steganographic method and its application for secure transmission of keyframes during wireless capsule endoscopy. Journal of medical systems, 40(5), p.114.

[3] Lian, S., 2008. Multimedia content encryption: techniques and applications. CRC press.

[4] Marwan, M., Kartit, A. and Ouahmane, H., 2016, May. A secure framework for medical image storage based on multi-cloud. In Cloud Computing Technologies and Applications (CloudTech), 2016 2nd International Conference on (pp. 88-94). IEEE.

[5] Laouamer, L., Nana, L.T., Al Shaikh, M. and Pascu, A.C., 2013, October. Informed symmetric encryption algorithm for DICOM medical image based on $\mathrm{N}$-grams. In Science and Information Conference (SAI), 2013 (pp. 353-357). IEEE.

[6] Mahmood, A., Dony, R. and Areibi, S., 2013, September. An adaptive encryption based genetic algorithms for medical images. In Machine Learning for Signal Processing (MLSP), 2013 IEEE International Workshop on (pp. 1-6). IEEE.

[7] Mahmood, A.B. and Dony, R.D., 2013, June. Adaptive encryption using pseudo-noise sequences for medical images. In Communications and Information Technology (ICCIT), 2013 Third International Conference on (pp. 39-43). IEEE.

[8] Mahmood, A., Hamed, T., Obimbo, C. and Dony, R., 2013. Improving the security of the medical images. Int J Adv Comput Sci Appl, 4(9).

[9] Puech, W. and Rodrigues, J.M., 2005, September. Crypto-compression of medical images by selective encryption of DCT. In Signal Processing Conference, 2005 13th European (pp. 1-4). IEEE.

[10] Ou, Y., Sur, C. and Rhee, K.H., 2007, August. Region-based selective encryption for medical imaging. In International Workshop on Frontiers in Algorithmics (pp. 62-73). Springer, Berlin, Heidelberg.

[11] Andrews, L.C. and Phillips, R.L., 2005. Laser beam propagation through random media (Vol. 152). Bellingham, WA: SPIE press.

[12] Patil, D.D. and Deore, S.G., 2013. Medical image segmentation: a review. International Journal of Computer Science and Mobile Computing, 2(1), pp.22-27.
[13] Pham, D.L., Xu, C. and Prince, J.L., 2000. Current methods in medical image segmentation. Annual review of biomedical engineering, 2(1), pp.315-337.

[14] Zheng, Y., Jeon, B., Xu, D., Wu, Q.M. and Zhang, H., 2015. Image segmentation by generalized hierarchical fuzzy C-means algorithm. Journal of Intelligent \& Fuzzy Systems, 28(2), pp.961-973.

[15] Gonzalez, R.C. and Woods, R.E., 2007. Image processing. Digital image processing, 2 .

[16] Lei, T., 2011. Statistics of medical imaging. CRC Press.

[17] C.W. Chen, J. Luo, K.J. Parker, "Image segmentation via adaptive Kmean clustering and knowledge based morphological operations with biomedical applications", IEEE Transactions on Image Processing, Vol.7 (12), 1998, pp 1673-1683

[18] Jaroš, M., Strakoš, P., Karásek, T., Říha, L., Vašatová, A., Jarošová, M. and Kozubek, T., 2017. Implementation of K-means segmentation algorithm on Intel Xeon Phi and GPU: Application in medical imaging. Advances in Engineering Software, 103, pp.21-28.

[19] Greenspan, H. and Pinhas, A.T., 2007. Medical image categorization and retrieval for PACS using the GMM-KL framework. IEEE Transactions on Information Technology in Biomedicine, 11(2), pp.190-202

[20] Pohle, R. and Toennies, K.D., 2001, July. Segmentation of medical images using adaptive region growing. In Medical Imaging 2001 (pp. 1337-1346). International Society for Optics and Photonics.

[21] Martinez-Perez, M., Hughes, A., Stanton, A., Thom, S., Bharath, A. and Parker, K., 1999. Retinal blood vessel segmentation by means of scale-space analysis and region growing. In Medical image computing and computer-assisted intervention-MICCAI'99 (pp. 90-97). Springer Berlin/Heidelberg.

[22] Kullback, S., 1968. Probability densities with given marginals. The Annals of Mathematical Statistics, 39(4), pp.1236-1243.

[23] DICOM. (2017). Part15. [ONLINE] Available at:http://dicom.nema.org/medical/dicom/current/output/pdf/part15.pdf [Accessed 25 May 2017].

[24] Stallings, W. (2010) Cryptography and network security: Principles and practice. 5th edn. Boston: Prentice Hall.

[25] Medicine.uiowa.edu. (2016). Visible Human Project CT Datasets Magnetic Resonance Research Facility. [ONLINE] Available at: https://medicine.uiowa.edu/mri/facilityresources/images/visiblehuman-project-ct-datasets [Accessed 15 November 2016]

[26] Healthcare.siemens.com. (2016). Magnetic Resonance Imaging Phoenix Gallery - Siemens Healthineers Global. [ONLINE] Available at: $\quad$ https://www.healthcare.siemens.com/magneticresonanceimaging/magnetom-world/clinical-corner/protocols/dicomimages[Accessed 15 November 2016].

[27] Osirix-viewer.com. (2016). OsiriX | DICOM Image Library [ONLINE] Available at:http://www.osirixviewer.com/resources/dicom-image-library/[Accessed 15 November 2016].

[28] The Cancer Imaging Archive (TCIA). (2016). The Cancer Imaging Archive (TCIA) - A growing archive of medical images of cancer. [ONLINE] Available at:http://www.cancerimagingarchive.net/[Accessed 15 November 2016].

[29] NEMA. (2017). DICOM dataset. [ONLINE] Available at:http://ftp://medical.nema.org/medical/Dicom/DataSets/[Accessed 29 April 2017].

[30] Farwa, S., Shah, T. and Idrees, L., 2016. A highly nonlinear S-box based on a fractional linear transformation. SpringerPlus, 5(1), p.1658.

[31] DICOM. (2017). Part15. [ONLINE] Available at:http://dicom.nema.org/medical/dicom/current/output/pdf/part15.pdf [Accessed 9 March 2018].

[32] Al-Haj, A., Abandah, G. and Hussein, N., 2015. Crypto-based algorithms for secured medical image transmission. IET Information Security, 9(6), pp.365-373. 